Received: 2017.01.26

Accepted: 2017.02 .15

Published: 2017.20.10

\section{Acute Chest Pain of Coronary Origin in Patients without Diabetes: How Multidetector Computed Tomography (MDCT) Angiography Helps in Diagnosis}

Authors' Contribution:
A Study Design
B Data Collection
C Statistical Analysis
D Data Interpretation
E Manuscript Preparation
F Literature Search
G Funds Collection

\author{
Moanes M. Enaba ${ }^{1 \mathrm{\Phi}}$, Ahmed A. Elsammak ${ }^{1 \mathrm{G}}$, Khaled M. Elgerby ${ }^{1 \mathrm{C}}$, Hatem M. Salem ${ }^{2 \varpi}$ \\ ${ }^{1}$ Department of Diagnostic Radiology, Faculty of Medicine, Zagazig University, Zagazig, Egypt \\ 2 Department of Internal medicine, Faculty of Medicine, Zagazig University, Zagazig, Egypt
}

Author's address: Moanes M. Enaba, Department of Diagnostic Radiology, Faculty of Medicine, Zagazig University, Zagazig, Egypt, e-mail: moanes_enaba@yahoo.com

\begin{abstract}
Background: $\quad$ To assess the diagnostic value of MDCT coronary angiography for evaluation of acute chest pain of coronary origin in non-diabetic patients.

Material/Methods: $\quad$ In 22 patients without DM, we assessed coronary arteries with multidetector CT angiography and invasive conventional angiography.

Results: CT coronary angiography revealed positive coronary artery disease findings in 16 patients; LAD was affected in $16(72.3 \%)$, RCA in $14(63.3 \%)$, and LCX in $8(36.4 \%)$ cases. The proximal part of LAD was the most commonly affected coronary artery (14 cases, $63.3 \%)$ in the studied patients who underwent MSCT coronary angiography. As regards the calcium score, 4 patients (18\%) had low calcium scores, 6 cases (27\%) had moderate calcium scores, and 12 cases (55\%) had high calcium scores. There were 6 patients with positive findings of coronary artery disease detected on MDCT coronary angiography that were not mirrored by conventional angiography.

Conclusions: $\quad$ MDCT angiography of the coronaries is a good and rapid method for evaluation of the coronary anatomy and for early detection and grading of coronary lesions in non-diabetic patients.

MeSH Keywords: $\quad$ Chest Pain • Coronary Angiography • Multidetector Computed Tomography

PDF file: $\quad$ http://www.polradiol.com/abstract/index/idArt/903536
\end{abstract}

\section{Background}

Acute chest pain is one of the most common reasons for patient visits in emergency departments. Patients are often worried about a potential heart attack, but there are many other causes of chest pain that should be considered; some of them are life threatening, while others are not [1].

Making correct decisions for patients with acute chest pain remains a challenge [2]

Diagnosis based on patient's medical history, initial cardiac enzyme levels, and initial electro-cardiograms (ECG) has many limitations [3].

Multiple chest problems, either vascular or non-vascular, including pulmonary embolism (PE), acute coronary syndromes, aortic dissection, pleural, pulmonary, or osseous lesions, must be taken into account [4].

Recently, contrast-enhanced multidetector computed tomography (MDCT) has become the imaging modality of choice, when a clinical suspicion of acute coronary syndrome or aortic dissection is raised. MDCT is capable of detecting, in a single scan, a multitude of non-threatening causes of acute chest pain, such as pneumonia, pericarditis, or fractures [5].

Recently, multiple studies have demonstrated that ECGgated CT of the whole chest is useful and allows for an accurate evaluation of the coronaries and non-vascular structures in a single scan, when a protocol called triple rule-out CT(TRO) is applied [6]. 


\section{Material and Methods}

\section{Patients}

A total of 22 patients (12 males) without diabetes mellitus (DM) who presented with acute chest pain of coronary origin were examined in Zagazig University hospitals between February 2016 and October 2016.

All patients underwent:

- Clinical assessment.

- Computed tomographic angiography.

- Coronary angiography.

\section{Selection of cases}

Patients were selected on the basis of:

- Clinical features of vascular origin: unexplained breathlessness (dyspnea) and/or tachypnea, hypotension, with or without pleuritic chest pain.

- Clinical examination: signs of acute chest pain, tachypnea (respiratory rate $>20 / \mathrm{min}$ ), tachycardia (heart rate $>100 / \mathrm{min})$, central cyanosis, fever, signs of acute right sided heart failure, hypotension, clinical signs of pulmonary hypertension, and presence of risk factors.

- Non-radiological data such as ECG changes suggestive of infarction (e.g., ST segment elevation or T wave inversion).

The exclusion criteria were:

- Contraindications to use contrast material (allergy, renal failure).

- Diabetes mellitus.

\section{Ethical consideration}

The protocol and informed consent forms used in this study were approved by the Institutional Review Board (IRB) of Zagazig University. All participants signed a written informed consent and filled a written survey consisting of demographic and clinical data.

\section{Methods}

\section{Clinical assessment:}

Full medical history:

- Personal history: name, age, sex, habits (e.g. smoking).

- Present history: character of acute chest pain, duration, site, radiation, exacerbating and relieving factors, associated symptoms, presence of a similar attack earlier, use of medication for a similar attack or for DM.

- Family history: DM, dyslipidemia, and blood disorders.

- Clinical examination: Performed by the referring physician.

- Laboratory investigations including: cardiac enzymes, renal function tests, blood glucose on admission, fasting blood glucose (FBG), postprandial blood glucose (after $2 \mathrm{~h}, 2 \mathrm{~h}-\mathrm{PPBG}$ ), and HBAlc (for excluding DM).

\section{Computed tomographic angiography}

A total of 22 patients without DM were enrolled for CT coronary angiography due to typical chest pain (defined as a retrosternal or precordial diffuse burning, heaviness, or squeezing sensation that may radiate to the left arm, neck or lower jaw, precipitated by effort and relieved by rest or nitrates); $73 \%$ of patients had intermediate risk of IHD, and about $27 \%$ had low risk of IHD.

The mean age was 55.5 years, ranging between 45 and 66 years. There were 12 males and 10 females. The average heart rate was 99 beats/min during the attack and 62 beats/ min during the scan.

Technique

Patient preparation:

- Fasting for 4-6 hours prior to the examination. Medications were not discontinued.

- Counseling: all steps of the procedure were explained in detail to the patients to evaluate their suitability for the scan, like breath-holding.

- Heart rate was evaluated before the examination. The examination was performed, if heart rate was below 65 beats per minute.

- Patients with heart rates above 70 beats per minute were given a cardioselective beta-blocker; $100 \mathrm{mg}$ of metoprolol or atenolol, orally 1 hour before the study, to obtain a stable low heart rate. Contra-indications to $\beta$-blockers were excluded.

\section{Scan protocol}

All examinations were performed with a 128 MDCT device (Philips) in the Radiology Department, Zagazig University hospital.

\section{Patient positioning and preparation for scanning}

- Supine position was used for CT examinations.

- ECG tracing was obtained with 3 ECG leads.

- Intravenous contrast injection with a flow rate of $5 \mathrm{~mL} / \mathrm{s}$.

- We asked patients for repeated test breath holds. Patients were advised to hold breath during inspiration, which was preceded by a cycle of inspiration and expiration.

\section{Image acquisition and reconstruction}

- Initially, a low-energy topogram was acquired.

- A bolus tracking technique was used to ensure homogeneous contrast enhancement of all coronaries, a series of dynamic low-dose axial scans (every 2 s) were taken at the level of the carina to track the bolus of contrast material and to monitor contrast enhancement at the level of the ascending aorta. When enhancement of 100120 Hounsfield units was reached, the MDCTA imaging sequence of the coronaries was started.

- Coronary CTA scan: sublingual nitroglycerin was administered about 3 minutes before the scan. If systolic blood pressure decreased below $100 \mathrm{~mm} \mathrm{Hg}$, nitroglycerin was not administered. The total volume of injected contrast agent ranged from 80 to $100 \mathrm{ml}$, and contrast infection was followed by a $50-\mathrm{mL}$ chaser of saline at a rate of $5 \mathrm{~mL} / \mathrm{s}$. A single breath-hold scan during comfortable inspiration was acquired. To obtain a volumetric CT dataset for the coronaries, the scan covered the whole 
heart from the proximal part of the ascending aorta (approximately $1-2 \mathrm{~cm}$ below the carina) to the diaphragmatic surface of the heart.

A 128-slice MDCT scanner was used to increase the volume coverage, reduce scan time, and decrease the amount of contrast agent. In addition, the scanner provided thinner slice collimation and faster tube rotation, improving both spatial and temporal resolution.

The whole procedure was completed in about fifteen minutes.

\section{Technical specifications of the imaging protocol for contrast- enhanced coronary angiography by MDCT}

\section{Coronary MDCT acquisition}

Cardiac CT scans were performed using 128-slice MDCT with the following parameters: slice thickness, $0.5 \mathrm{~mm}$; helical retrospective gating and reconstruction interval of $1 \mathrm{~mm}$.

\section{Specification 128-slice scanner}

- Tube current (mAs), 340.

- Tube voltage $(\mathrm{kV}), 120$.

- Tube rotation time (ms), 280.

- Scan range (mm), 153.

- Contrast dose (mL), 80 .

\section{Image reconstruction}

CTA angiography of the coronaries was performed in the spiral acquisition mode, with continuous acquisition of data throughout the cardiac cycle. To achieve a good quality of the reconstructed axial images, we used the following parameters: slice thickness, $0.75 \mathrm{~mm}$; pixel matrix, $512 \times 512$; increment between images, $0.4 \mathrm{~mm}$; thinner slices increased the resolution of the 3-D dataset and improved the quality of reformatted images; we used a small field of view (FOV), 18 to $20 \mathrm{~cm}$. A larger FOV of $35 \mathrm{~cm}$ was used only if there were incidental extracardiac findings.

\section{Image evaluation}

The axial images were most important for the evaluation, as they can reveal all recognized pathologies, and multiplanar reformatted (MPR) images can visualize the coronaries in multiple orientations, thereby showing the presence of significant stenosis in the coronaries.

After detecting calcified and non-calcified plaques on axial images, 3 to $5 \mathrm{~mm}$, thin-slab, maximum intensity projection (MIP) images were obtained to project the information of 4 to 7 original slices into one image.

The degree of luminal narrowing was evaluated by comparing the narrowed segment lumen with proximal and distal normal lumens, with manual correction or placement of the calipers and reconstruction of a true cross-sectional image of the vessel orthogonal to the long-axis view.
Table 1. Clinical examination of the studied patients who underwent MSCT coronary angiography.

\begin{tabular}{lcc}
\hline \multirow{2}{*}{ Clinical examination } & \multicolumn{2}{c}{ Patients (N=22) } \\
\cline { 2 - 3 } & No. & $\%$ \\
\hline Heart rate (beats/min) & & \\
\hline Average & 10 & $45.5 \%$ \\
\hline Tachycardia & 12 & $54.5 \%$ \\
\hline Pulse rate & & \\
\hline Average & 10 & $45.5 \%$ \\
\hline Rapid & 12 & $54.5 \%$ \\
\hline Pulse volume & & \\
\hline Average & 16 & $72.7 \%$ \\
\hline Weak & 6 & $27.3 \%$ \\
\hline Respiratory rate & & \\
\hline Average & 20 & $90.9 \%$ \\
\hline Tachypnea & 2 & $9.1 \%$ \\
\hline Blood pressure & 12 & $54.5 \%$ \\
\hline Normotensive & 10 & $45.5 \%$ \\
\hline Hypertensive & & \\
\hline
\end{tabular}

A quantitative assessment could be made in some cases that had excellent image quality and no severe calcifications; this improved diagnostic accuracy for the detection of significant stenoses. Direct measurements of lumen caliber were used particularly for intermediate lesions.

Curved MPR images, curved MIP images, and 3-D volumerendering technique images were used to provide a general overview of the coronary and cardiac anatomy.

\section{Statistical analysis}

All data were collected, tabulated, and statistically analyzed using the SPSS 19.0 software, for Windows (SPSS Inc., Chicago, IL, USA) and MedCalc 13 software, for Windows (MedCalc Software bvba, Ostend, Belgium). Quantitative data were expressed as means \pm SDs and medians (range), and qualitative data were expressed as absolute frequencies (number) and relative frequencies (percentage). $\mathrm{P}<0.05$ was considered statistically significant $(\mathrm{S}), \mathrm{p}<0.001$ was considered highly statistically significant (HS), and $\mathrm{p} \geq 0.05$ was not considered statistically significant (NS).

\section{Results}

Based on the clinical examination of patients without DM, a large number of patients had tachycardia, rapid pulse rate, average pulse volume, average respiratory rate, and normal blood pressure (Table 1). 
Table 2. ECG findings of the studied patients who underwent MSCT coronary angiography.

\begin{tabular}{lcc}
\hline \multirow{2}{*}{ ECG finings } & \multicolumn{2}{c}{ Patients (N=22) } \\
\cline { 2 - 3 } & No. & $\%$ \\
\hline Non-specific & 12 & $54.5 \%$ \\
\hline Specific & 10 & $45.5 \%$ \\
\hline Depression of ST segment & 4 & $27.3 \%$ \\
\hline T wave changes & 6 & $18.1 \%$ \\
\hline
\end{tabular}

Table 3. Laboratory findings of the studied patients who underwent MSCT coronary angiography.

\begin{tabular}{|c|c|c|}
\hline \multirow{2}{*}{ Laboratory findings } & \multicolumn{2}{|c|}{ Patients ( $N=22)$} \\
\hline & No. & $\%$ \\
\hline \multicolumn{3}{|l|}{ Cardiac enzymes } \\
\hline Normal & 22 & $100 \%$ \\
\hline Abnormal & 0 & $0 \%$ \\
\hline \multicolumn{3}{|l|}{ D-dimer (ng/mL) } \\
\hline Normal & 18 & $81.8 \%$ \\
\hline Abnormal & 4 & $18.2 \%$ \\
\hline Mean \pm SD & \multicolumn{2}{|c|}{$306.81 \pm 97.88$} \\
\hline Median (range) & \multicolumn{2}{|c|}{$265(250-505)$} \\
\hline \multicolumn{3}{|l|}{ Cholesterol (mg/dl) } \\
\hline Normal & 2 & $9.1 \%$ \\
\hline Abnormal & 20 & $90.9 \%$ \\
\hline Mean $\pm S D$ & \multicolumn{2}{|c|}{$274.36 \pm 59.27$} \\
\hline Median (range) & \multicolumn{2}{|c|}{$255(199-400)$} \\
\hline
\end{tabular}

ECG findings were non-specific in 12 patients, a depressed ST segment was found in 4 patients, and $\mathrm{T}$ wave changes were seen in 6 patients (Table 2).

As regards laboratory findings, a high number of patients had elevated cholesterol, LDL, HDL, normal cardiac enzymes, and D- dimmer (Table 3).

CT coronary angiography revealed that 16 cases had positive coronary artery disease findings; LAD was affected in $16(72.3 \%)$ cases, RCA in 14 (63.3\%) cases, and LCX in 8 (36.4\%) cases (Table 4).

The proximal part of LAD was the most commonly affected coronary artery in the studied patients who underwent MSCT coronary angiography (Table 5)
Table 4. MSCT coronary angiography findings of the studied patients.

\begin{tabular}{lcc}
\hline \multirow{2}{*}{$\begin{array}{c}\text { MSCT coronary angiography } \\
\text { findings }\end{array}$} & \multicolumn{2}{c}{ Patients (N=22) } \\
\cline { 2 - 3 } Coronary artery stenosis & No. & $\%$ \\
\hline Absent & 6 & $27.3 \%$ \\
\hline Present & 16 & $72.7 \%$ \\
\hline Involved vessel & & \\
\hline LAD & 16 & $72.7 \%$ \\
\hline RCA & 14 & $63.6 \%$ \\
\hline LCX & 8 & $36.4 \%$ \\
\hline
\end{tabular}

\begin{tabular}{|c|c|c|}
\hline \multirow{2}{*}{ Laboratory findings } & \multicolumn{2}{|c|}{ Patients ( $N=22)$} \\
\hline & No. & $\%$ \\
\hline \multicolumn{3}{|l|}{$\mathrm{LDL}(\mathrm{mg} / \mathrm{dl})$} \\
\hline Normal & 2 & $9.1 \%$ \\
\hline Abnormal & 20 & $90.9 \%$ \\
\hline Mean $\pm S D$ & \multicolumn{2}{|c|}{$168.45 \pm 29.26$} \\
\hline Median (range) & \multicolumn{2}{|c|}{$180(90-190)$} \\
\hline \multicolumn{3}{|l|}{$\mathrm{HDL}(\mathrm{mg} / \mathrm{dl})$} \\
\hline Normal & 2 & $9.1 \%$ \\
\hline Abnormal & 20 & $90.9 \%$ \\
\hline Mean \pm SD & \multicolumn{2}{|c|}{$47.36 \pm 8.86$} \\
\hline Median (range) & \multicolumn{2}{|c|}{$50(35-63)$} \\
\hline \multicolumn{3}{|l|}{ Arterial blood gases } \\
\hline Normal & 22 & $100 \%$ \\
\hline Abnormal & 0 & $0 \%$ \\
\hline \multicolumn{3}{|l|}{ FBG, 2h-PPBG, HBA1c } \\
\hline Normal & 22 & $100 \%$ \\
\hline Abnormal & 0 & $0 \%$ \\
\hline
\end{tabular}

As regards the calcium score, 4 cases had low calcium scores, 6 cases had moderate calcium scores, and 12 cases had high calcium scores (Table 6).

As regards the comparison between MDCT, coronary angiography, and conventional coronary angiography, MDCT coronary angiography detected positive findings in 6 patients that were not mirrored by conventional angiography (Table 7; Figures 1-3). 
Table 5. Affected coronary arteries in the studied patients who underwent MSCT coronary angiography.

\begin{tabular}{lcccc}
\hline \multirow{2}{*}{ Affected coronary artery } & \multicolumn{2}{c}{ Patients (N=22) } & \multicolumn{2}{c}{ Extent of involvement (\%) } \\
\cline { 2 - 5 } & No. & $\%$ & Mean \pm SD & Median (range) \\
\hline LAD involvement & & & & \\
\hline Proximal & 14 & $63.6 \%$ & $61.71 \pm 18.71$ & $66(40-90)$ \\
\hline Mid & 8 & $36.4 \%$ & $57.50 \pm 15$ & $50(50-80)$ \\
\hline Distal & 2 & $9.1 \%$ & 50 & $45(45-70)$ \\
\hline RCA involvement & & & & $32(25-53)$ \\
\hline Proximal & 6 & $27.3 \%$ & $53.33 \pm 14.43$ & $35.50 \pm 12.23$ \\
\hline Mid & 8 & $36.4 \%$ & & $50(34-70)$ \\
\hline LCX involvement & 8 & $36.4 \%$ & $51 \pm 15.29$ & \\
\hline Proximal & & & & \\
\hline
\end{tabular}

Table 6. Calcium scores of the studied patients.

\begin{tabular}{lcc}
\hline \multicolumn{1}{c}{ Calcium score } & N=22 & $\%$ \\
\hline No evidence of CAD & 0 & 0 \\
\hline Very low 1-10 & 0 & 0 \\
\hline Low 11-100 & 4 & 18.2 \\
\hline Moderate 101-400 & 6 & 27.3 \\
\hline High $>400$ & 12 & 54.6 \\
\hline
\end{tabular}

\section{Discussion}

In this study, a large number of patients presented to our emergency department due to acute chest pain suggestive of acute coronary syndrome. Some of them had myocardial infarction, as evidenced by typical chest pain, ST segment elevation, and elevated initial cardiac biomarkers. These patients were referred directly to a cardiology department for PCI or thrombolysis, whilst others were referred to a radiology department for CT coronary angiography. However, initial non-contrast cardiac CT revealed high calcium scores (more than 1000) and rendered CT coronary angiography unnecessary for some patients, other patients were found unfit for the exam. A total of 22 patients without DM (based on ADA diagnostic criteria for DM, 2016) [7] presented with acute chest pain, non-diagnostic resting
ECG and echocardiography, and normal cardiac biomarkers, and they were enrolled for multidetector CT angiography of the coronary arteries between February 2016 and October 2016.

The mean age of patients was 55.5 years, ranging from 45 to 66 years. Twelve $(54.5 \%)$ patients were male, and 10 $(45.5 \%)$ patients were female.

As regards the prevalence of risk factors, there is an agreement between our study and a study performed in 2015 in 20 patients with acute chest pain and non-diagnostic resting ECG and echocardiography, and normal cardiac biomarkers. These patients were enrolled for multidetector CT angiography of the coronaries, and it was found that $10(45.45 \%)$ cases were hypertensive, $6(27.27 \%)$ cases were smokers, and 20 (90.9\%) cases had dyslipidemia. We found that $12(54.54 \%)$ cases had positive family history and 12 $(54.54 \%)$ cases were obese. As regards the calcium score, similarly to our study, it was found to be below 800 in all the examined patients [8]

In this study, 6 (27.27\%) cases had normal coronary arteries, $6(27.27 \%)$ cases showed non-significant CAD, 10 $(45.45 \%)$ cases showed significant $\mathrm{CAD}$, and $2(9 \%)$ showed myocardial bridges. In a study carried out in 2007, that enrolled 58 participants with a history of acute chest pain, $15(28.8 \%)$ cases had no detectable coronary artery lesions on CT angiography, 20 (34\%) patients had nonsignificant lesions, and $23(39 \%)$ had significant stenoses [9].

Table 7. Agreement between MSCT coronary angiography and conventional angiography.

\begin{tabular}{llcccc}
\hline & & \multicolumn{2}{c}{ Conventional angiography } & Total \\
\cline { 2 - 5 } & & + ve & -ve (or less) & 16 \\
\hline \multirow{2}{*}{$\begin{array}{l}\text { MSCT coronary } \\
\text { angiography }\end{array}$} & +ve & 10 & 6 & 6 \\
\hline Total & & 0 & 6 & 22 \\
\hline
\end{tabular}

P value $<0.05$ was considered statistically significant. 


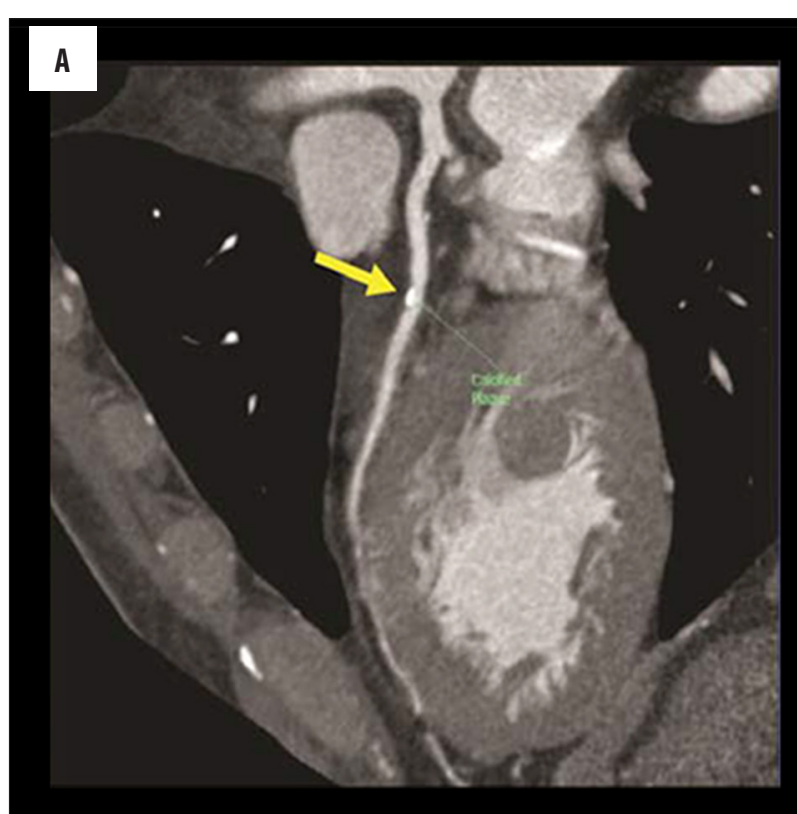

\section{B}
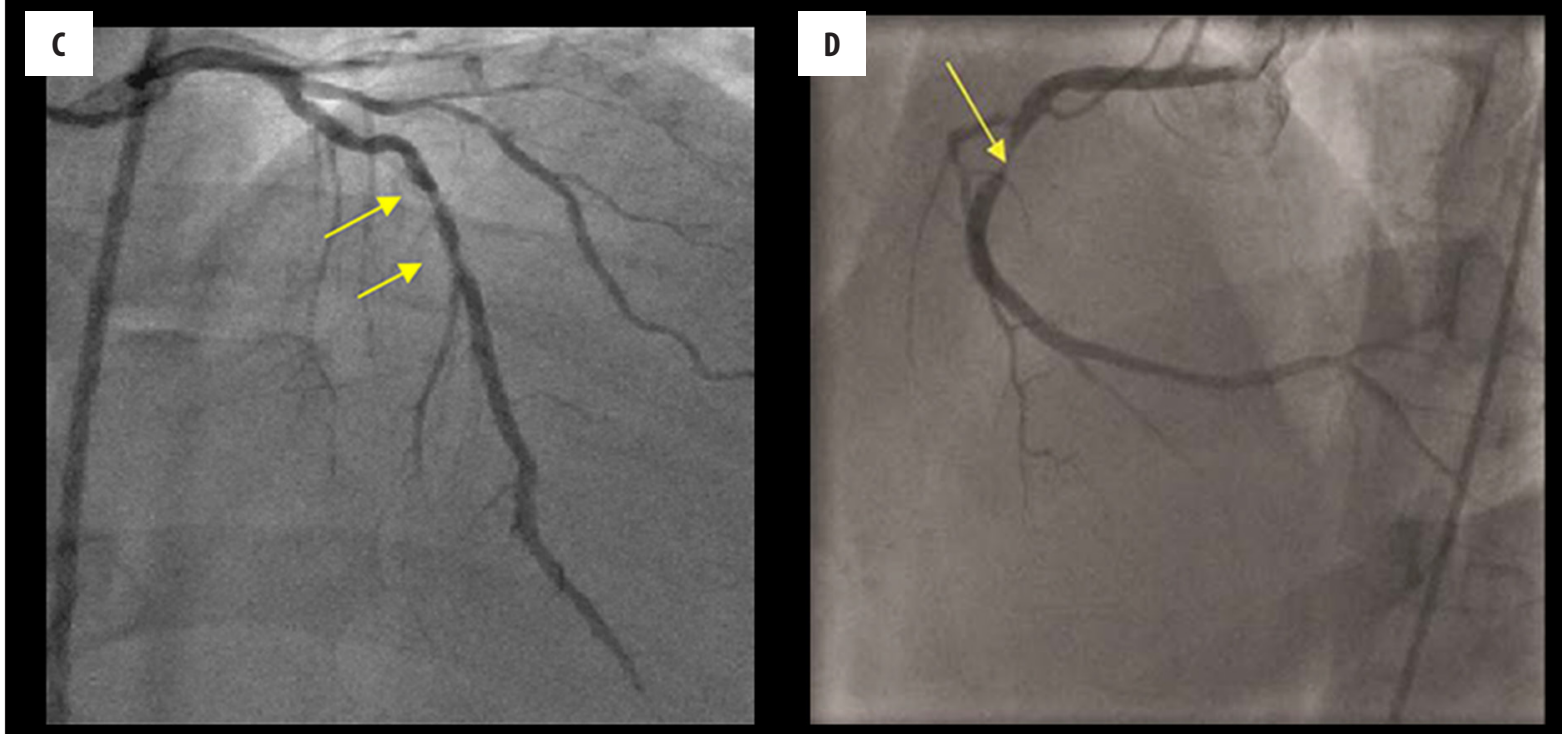

Figure 1. Male patient, a 55-year-old smoker, non-diabetic, with hyperlipidemia and complaints of retrosternal acute chest pain (A), (B) CPR image of LAD shows proximal and middle segments with multiple calcified (yellow arrow) and mixed (green arrow) plaques and mild to moderate stenotic lesions (maximum 50\% stenosis), (C), (D) Invasive coronary angiography shows LAD, confirming the proximal and middle segment stenosis(arrows).

Plaques were seen in $16(72.72 \%)$ patients, $14(87.5 \%)$ of them had calcified plaques, 10 patients $(62.5 \%)$ had noncalcified plaques, and 6 (37.5\%) of them had mixed plaques.

As regards the affected vessels, we found that the left anterior descending artery (LAD) was affected in $16(72.7 \%)$ cases, the left circumflex artery was affected in 8 (36.4\%) cases, and RCA was affected in $14(63.6 \%)$ cases, which is agreement with a study performed in 2014 that included 50 patients referred for MSCT coronary angiography (patients with previous coronary bypass grafts and those with coronary stents were excluded). History of contrast allergy, renal impairment, and severe chest conditions were the remaining exclusion criteria. Coronary angiographic CT studies were performed with a 320 CT scanner and determined that the most common site of stenosis was the left anterior descending artery (76.7\%), but the circumflex artery $(43.3 \%)$ and the right or left main coronary arteries $(40 \%)$ were affected differentially, when compared to our study [10].

As regards the number of affected vessels, 6 cases $(27.2 \%)$ had normal coronary arteries, one vessel was affected in 2 cases $(9.1 \%)$, two vessels were affected in 6 cases $(27.3 \%)$, and three vessels were affected in 8 cases (36.4\%), which shows that multi-vessel disease is most common. This is not in agreement with a study performed in 2015 in which $35 \%$ of cases had one affected vessel, $30 \%$ had two affected vessels, and $5 \%$ had 3 affected vessels [8]. 


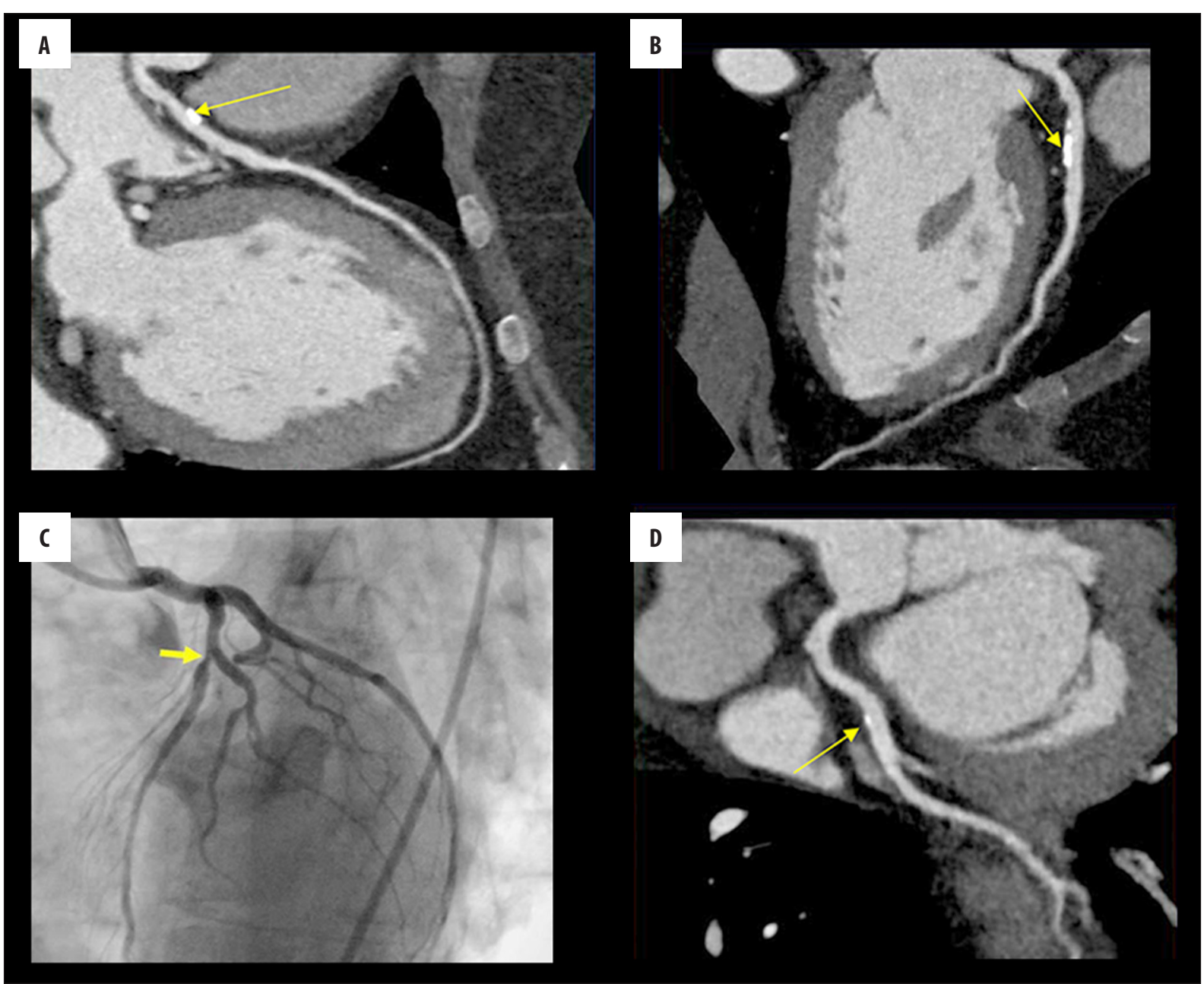

Figure 2. Female patient, 63 years of age, with uncontrolled hyperlipidemia and complaints of retrosternal acute chest pain (A), (B) CPR image of LAD shows that the proximal segment has a calcified plaque (about $15 \mathrm{~mm}$ in length) that causes approximately $40 \%$ luminal stenosis. The diagonal branches have no stenoses or calcified plaques. The distal LAD shows preserved caliber up to the cardiac apex. (C) Invasive conventional coronary angiography of $L A D$ confirms the degree of stenosis (40\%, arrow), (D) CPR image of $L C X$, the proximal left circumflex artery has a calcified mural plaque. No significant stenosis or non-calcified plaques were noted.

Findings of CTCA are correlated with invasive conventional coronary angiography with a concordance rate of $72.7 \%$. There is an agreement in sensitivity and NPV (both 100\%) in studies performed in 2007 and 2014 with 64 MDCT and 320 MDCT, respectively $[9,10]$.

In our study, patients with pre-examination heart rate above 65 beats per minute were given a cardioselective B-blocker (orally 1 hour before the study) to obtain a stable low heart rate, if no contra-indications to B-blockers were present. If heart rate was still above 75 beats per minutes, the examination was postponed. This is in agreement with the protocol of Pugliese et al., who reported that the examination should be postponed, if heart rate is above 75 beats/ $\min [8]$.

Similarly, nitroglycerine was not administrated prior to the study, because, in spite of its coronary-dilator effect that enhance vessel visualization, the drug can increase heart rate and lead to overestimation of diameter reduction of stenotic lesions, as nitroglycerine dilates the healthy segments [8].
As regards image quality in relation to the cardiac cycle, the left main and LDA arteries were best visualized in middiastole (at $60-70 \%$ ) in most patients. For the right coronary artery, the optimal timing of acquisition was in diastole (at $40 \%$ ). The left circumflex artery showed optimal image quality at $50-60 \%$. This is in agreement with a study from 2001 that reached the same conclusions [11].

The visibility of a coronary artery segment was considered good, when there was a sharp delineation from the surrounding structures, a nearly artifact-free course of the segment, with little blurring even in its peripheral sections, and sufficient contrast between the vessel lumen and wall. Visualization was considered adequate in the presence of image degrading artifact that allowed evaluation with moderate confidence, and it was considered poor in the presence of image degrading artifacts (evaluation was possible but only with low confidence). The examined segment was considered non-assessable, when image degrading artifacts were severe enough to prevent differentiation between significant stenosis and occlusion or between normal segments and mildly atherosclerotic lesions [11]. 


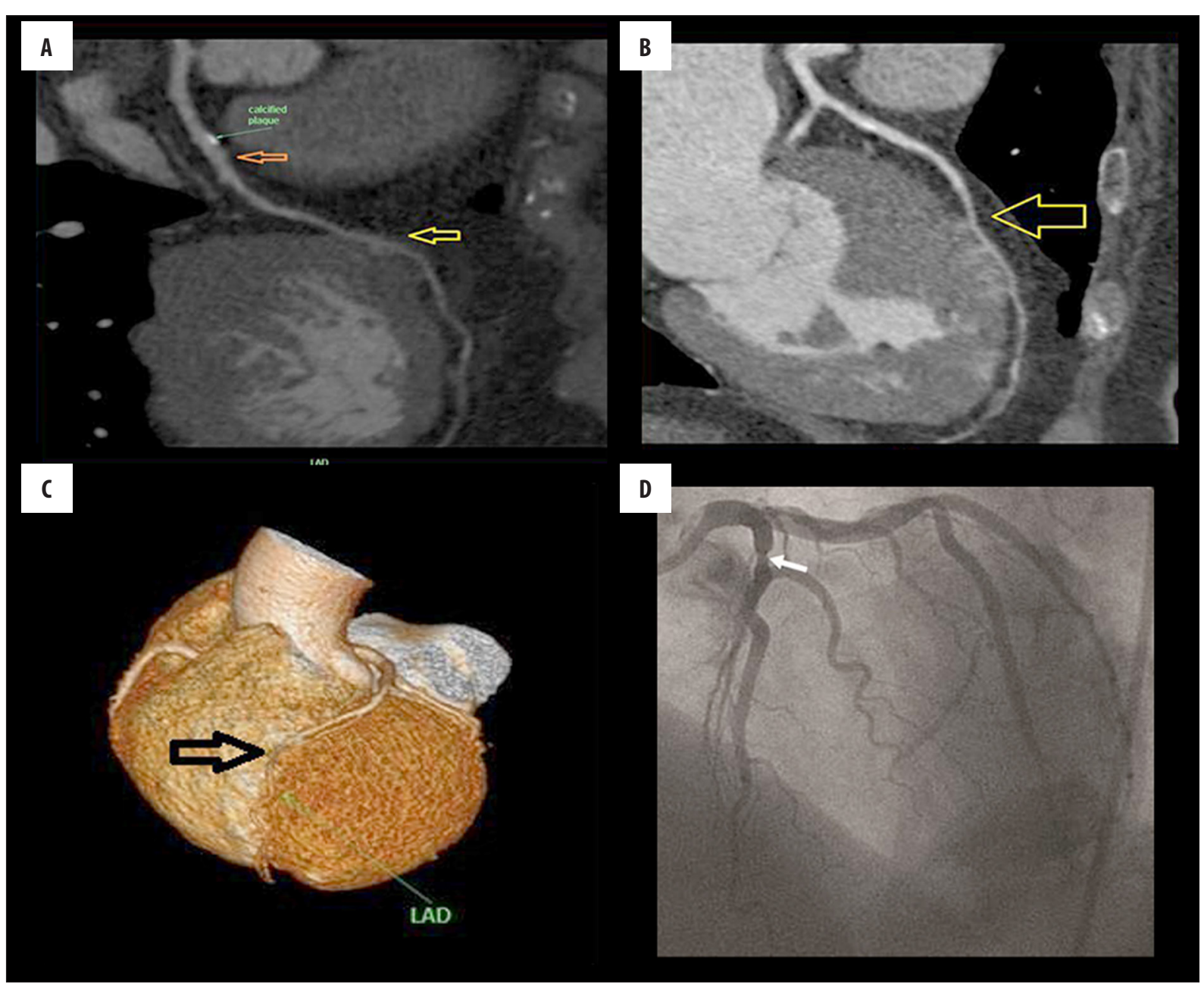

Figure 3. Female patient, 55 years of age, hypertensive, with hyperlipidemia and complaints of retrosternal acute chest pain associated with syncope, dyspnea, and tachycardia (A) CPR image of LAD shows that the proximal segment has a small, calcified plaque (located about $27 \mathrm{~mm}$ from the origin of the artery, orange arrow). It measures about $3 \mathrm{~mm}$ in length and causes up to $41 \%$ (non-significant) stenosis; the middle segment shows adequate contrast filling with a segment (19 mm long) of myocardial bridging (yellow arrow); thickness of covering myocardium $=4 \mathrm{~mm}$; the distal segment gradually decreases in caliber, wrapping around the cardiac apex, (B) CPR image of LAD shows a myocardial bridge over the middle LAD segment (arrow), (C) VR 3D reconstruction of LAD confirms the presence of myocardial bridge (black arrow), (D) Invasive coronary angiography of LAD shows proximal segment stenosis of $40 \%$ (arrow).

It is now clear that non-invasive CT angiography of the coronary arteries requires the use of at least 16-slice scanners, with improved imaging observed in 64-slice scanners. In this study, a 128-slice CT scanner showed improved sensitivity and specificity in comparison to 64-slice scanners; this could be attributed to reduction of tube rotation time with consequent improvement in temporal resolution [12].

\section{Conclusions}

Based on the results of our study, we concluded that MDCT coronary angiography is a good and rapid non-invasive imaging modality that is able to evaluate the coronary anatomy and detect and grade early coronary lesions in non-diabetic patients.

\section{References:}

1. Tintinalli's Emergency Medicine. A Comprehensive Study Guide. $7^{\text {th }}$ edition. McGraw-Hill, 2010; 491-524

2. Frauenfelder T, Appenzeller P, Karlo C et al: Triple rule-out Ct in the emergency department: protocols and spectrum of imaging findings. Eur Radiol, 2009; 19(4): 789-99

3. Goodacre S, Locker T, Morris F et al: How useful are clinical features in the diagnosis of acute, undifferentiated chest pain? Acad Emerg Med, 2002; 9: 203-8
4. Feuchtner G, Postel T, Weidinger $\mathrm{F}$ et al: Is there a relation between non-calcifying coronary plaques and acute coronary syndromes? A retrospective study using multi-slice computed tomography. Cardiology, 2008; 110(4): 241-48

5. Hayter RG, Rhea JT, Small A et al: Suspected aortic dissection and other aortic disorders: Multi-detector row CT in 373 cases in the emergency setting. Radiology, 2006; 238: 841-52

6. Johnson TR, Nikolaou K, Becker A et al: Dual-source CT for chest pain assessment. Eur Radiol, 2008; 18: 773-80

7. American Diabetes Association: Standards of medical care in diabetes. Diabetes Care, 2016; 39(Suppl. 1): S16 
8. Kassem Hanan K, Dabees Naglaa L, El-Sheikh Ayman A et al: Multislice CT coronary angiography is an additional tool for provisional diagnosis of acute chest pain with neither ECG nor echocardiography significant findings in emergency room. Tanta Medical Journal, 2015; 43: 113-19

9. Rubinshtein R, Halon D, Gaspar T et al: Usefulness of 64-slice cardiac computed tomographic angiography for diagnosing acute coronary syndromes and predicting clinical outcome in emergency department patients with chest pain of uncertain origin. Circulation, 2007; 115: 1762-68
10. Youssef MA, Dawoud MA, Elbarbary AA et al: Role of 320-slice multislice computed tomography coronary angiography in the assessment of coronary artery stenosis. Egyptian Society of Radiology and Nuclear Medicine, 2014; 45(2): 317-24

11. Kopp AF, Schroeder S, Kuettner A et al: Coronary arteries: retrospectively ECG-gated multi-detector row CT angiography with selective optimization of the image reconstruction window. Radiology, 2001; 221: 683-88

12. Lepor NE and Madyoon H: The emerging use of 16-and 64-slice computed tomography coronary angiography in clinical cardiovascular practice. Rev Cardiovasc Med, 2005; 6(1): 47-53 